\title{
Is there evidence for neurodegenerative change following traumatic brain injury in children and youth? A scoping review
}

\section{Michelle L. Keightley ${ }^{1,2,3,4,5 *}$, Katia J. Sinopoli ${ }^{1,6}$, Karen D. Davis ${ }^{78}$, David J. Mikulis ${ }^{7}$, Richard Wennberg ${ }^{9}$, Maria C. Tartaglia ${ }^{9}$, Jen-Kai Chen ${ }^{10}$ and Charles H. Tator ${ }^{9}$}

${ }^{1}$ Bloorview Research Institute, Holland Bloorview Kids Rehabilitation Hospital, Toronto, ON, Canada

${ }^{2}$ Department of Occupational Science and Occupational Therapy, University of Toronto, Toronto, ON, Canada

${ }^{3}$ Graduate Department of Rehabilitation Science, University of Toronto, ON, Canada

${ }^{4}$ Department of Psychology, University of Toronto, ON, Canada

${ }^{5}$ Cognitive Neurorehabilitation Sciences, Toronto Rehabilitation Institute, Toronto, ON, Canada

${ }^{6}$ Department of Psychology and Division of Neurology, Sickids Hospital for Sick Children, Toronto, ON, Canada

7 Division of Brain, Imaging and Behaviour - Systems Neuroscience, Toronto Western Research Institute, University Health Network, Toronto, ON, Canada

${ }^{8}$ Department of Surgery and Institute of Medical Science, University of Toronto, Toronto, ON, Canada

${ }^{9}$ Krembil Neuroscience Centre, Toronto Western Hospital, University Health Network and University of Toronto, Toronto, ON, Canada

${ }^{10}$ Neuropsychology/Cognitive Neuroscience Unit, Montreal Neurological Institute, Montreal, OC, Canada

\section{Edited by:}

James Blair, National Institute of Mental Health, USA

\section{Reviewed by:}

Jack Van Honk, Utrecht University, Netherlands

John Van Horn, University of

California at Los Angeles, USA

*Correspondence:

Michelle L. Keightley, Bloorview Research Institute, Holland

Bloorview Kids Rehabilitation

Hospital, 150 Kilgour Rd., Toronto,

ON M4G 1R8, Canada

e-mail:mkeightley@

hollandbloorview.ca

While generalized cerebral atrophy and neurodegenerative change following traumatic brain injury (TBI) is well recognized in adults, it remains comparatively understudied in the pediatric population, suggesting that research should address the potential for neurodegenerative change in children and youth following TBI. This focused review examines original research findings documenting evidence for neurodegenerative change following TBI of all severities in children and youth. Our relevant inclusion and exclusion criteria identified a total of 16 articles for review. Taken together, the studies reviewed suggest there is evidence for long-term neurodegenerative change following TBI in children and youth. In particular both cross-sectional and longitudinal studies revealed volume loss in selected brain regions including the hippocampus, amygdala, globus pallidus, thalamus, periventricular white matter, cerebellum, and brain stem as well as overall decreased whole brain volume and increased CSF and ventricular space. Diffusion Tensor Imaging (DTI) studies also report evidence for decreased cellular integrity, particularly in the corpus callosum. Sensitivity of the hippocampus and deep limbic structures in pediatric populations are similar to findings in the adult literature and we consider the data supporting these changes as well as the need to investigate the possibility of neurodegenerative onset in childhood associated with mild traumatic brain injury (mTBI).

\section{Keywords: traumatic brain injury, neurodegeneration, magnetic resonance imaging}

\section{INTRODUCTION}

One of the most commonly reported injuries in children who participate in sports is concussion or mild traumatic brain injury (mTBI) (Browne and Lam, 2006). Children and youth $(<19$ years) involved in organized contact sports are nearly six times more likely to suffer a severe concussion compared to other leisure physical activities (Browne and Lam, 2006). The recovery profile and breadth of consequences in children and youth remains largely unknown (McCrory et al., 2004). This dearth of literature is compounded by the recent scrutiny youth participation in competitive contact sports (such as boxing, hockey and football) has received, due primarily to case study and media reports linking repeat concussions to a distinct neurodegenerative disease known as chronic traumatic encephalopathy (CTE).

This condition was initially described in boxers in 1928 by Martland and known as dementia pugilistica (Martland, 1928). In some cases, a constellation of symptoms typical of neurodegenerative disease were observed in a syndrome, and Miller coined the term "CTE" (Miller, 1966). It is now recognized in many sports in which there are repetitive concussions. CTE is defined as a slowly progressive neurodegenerative disorder associated with repeated brain trauma that manifests years after implicated concussive events (McKee et al., 2009). CTE is a neurodegenerative disease with a distinct distribution of atrophy along the amygdalo-hippocampal-septo-hypothalamicmesencephalic continuum (McKee et al., 2009). CTE shows some similarity to the chronic effects of moderate and severe traumatic brain injury (TBI). There is demonstrated evidence for neurodegeneration in the chronic phase of moderate to severe TBI, ensuing months to years after brain injury with sub-acute atrophy within the limbic system hippocampi (Ng et al., 2008) and elsewhere (Greenberg et al., 2008; Farbota et al., 2012; Green et al., 2014). The corpus callosum (unmyelinated axons in particular) is vulnerable to the deposition of protein post-TBI, suggesting 
commonality with CTE (Reeves et al., 2007). Thus, generalized cerebral atrophy is a well-established consequence of moderateto-severe TBI in adults that can be quantified from MRI studies that assess total brain volume (e.g., Bigler et al., 2010).

To the best of our knowledge, there are currently no scientific studies published of CTE following repetitive concussions/mTBI in children. The popularity of competitive sports coupled with the dearth of literature investigating long-term outcomes following $\mathrm{mTBI}$ in the pediatric population, suggests that research addressing the potential for CTE in youth following multiple mTBIs should be a public priority. This mini-review examines the available evidence on atrophy and neurodegenerative change in children and adolescents ( $<19$ years) in the chronic stages of mild, moderate and severe TBI compared to typically developing youths. Research findings describe widespread volume reductions (e.g., Levin et al., 2000; Verger et al., 2001; Serra-Grabulosa et al., 2005; Tasker et al., 2005; Wilde et al., 2005, 2006, 2007, 2010, 2012; Braga et al., 2007; Spanos et al., 2007; Yuan et al., 2007; Fearing et al., 2008; Bigler et al., 2010; Beauchamp et al., 2011a,b) and clearly indicate that childhood TBI disrupts normal agerelated neuronal processes that may persist across the life-span (see Bigler, 2013).

\section{METHODS}

\section{IDENTIFYING RELEVANT STUDIES}

We chose a scoping review methodology (Mays et al., 2001) and entered the keywords mild, traumatic, brain, injury, MRI, child, chronic, long-term, and concussion combined with the Boolean operators AND and OR into PubMed, Ovid, PsychInfo, and Medline as the search engines. We also hand searched each reference list and included only published articles from January 1, 2000 to May 2, 2012 that contained human participants and were published in English. The start date of 2000 was chosen as studies published following this date contained imaging technology and methods sufficiently advanced in terms of sensitivity to detect more subtle structural changes. Foreign language material was excluded because of the cost and time in translating material. We adopted these methods for practical reasons and acknowledge that key articles may have been missed.

The various mechanisms for searching in our scoping study generated a total of 16 publications. No additional publications were identified as the study progressed. All publications were originally identified on the PubMed electronic databases and confirmed by subsequent databases searched.

\section{STUDY SELECTION}

Our initial examination of the studies indicated that our search strategy had identified a large number of irrelevant studies. Criteria to eliminate studies that did not address our central research question were developed post-hoc in three stages, based on increasing familiarity with the literature (Arksey and O'Malley, 2005). In Stage One we included original research articles and case studies that examined structural changes using MRI following mild, moderate and severe TBI. We included imaging studies examining adults only if the methods informed imaging techniques that could be applied to pediatric cases. We excluded meta-analyses and review articles as well as non-TBI forms of brain injury. These criteria identified 201 articles for review. During Stage Two, we narrowed our inclusion criteria to a TBI sustained during childhood and youth (defined as under the age of 19) and excluded metabolic studies which identified 71 articles. Finally during Stage Three we included only those studies that were cross-sectional or longitudinal in design and reported on neuroimaging findings of neurological degeneration obtained at least 1 year post-injury in order to identify those studies focused on the chronic effects following TBI for all subjects examined. We also excluded studies focusing solely on intentional brain trauma (i.e., inflicted abuse) to try and keep injury mechanism more similar to the biomechanical forces observed in concussion/mTBI. These criteria resulted in 16 articles for review.

Two reviewers (first and second authors) applied the inclusion and exclusion criteria to all the citations and copies of the full articles were obtained for those studies felt to "best fit" the research question. Having read the articles in full, all 16 articles were selected for inclusion in the review.

\section{CHARTING THE DATA}

We charted key items of information obtained from the primary research reports being reviewed (Arksey and O'Malley, 2005). We recorded information as follows:

- Author(s), year of publication and study location

- Study population (Brain Injury (TBI) Severity, Time Since Injury, and Mechanism)

- Study Population (Demographic Characteristics)

- Aims

- Study Design

- Structural Feature Assessed

- Behavioral Outcome Measure(s)

- Neurodegenerative Findings

\section{RESULTS}

NUMERICAL ANALYSIS OF THE EXTENT, NATURE, AND DISTRIBUTION OF STUDIES

\section{Study design}

Supplementary Table 1 summarizes the data obtained from each study. With respect to study design, 14 of the 16 studies reviewed utilized a cross-sectional design, of which 10 included a comparison group. Of the 10 studies containing a control group, seven studies individually matched participants across a number of demographic variables including age, sex, education (maternal or child), and socioeconomic status. One study matched on age and sex combined, with one study matching on age alone. Two studies described a control group consisting of children and youth of similar age who had sustained orthopedic injuries. All cross sectional studies included participants who were under the age of 19 years at the time of scanning. Two of the sixteen studies were prospective longitudinal investigations of the same cohort of children re-imaged at two time-points. One study re-imaged at 3 and 18 months post-injury while the second re-imaged at 3 and 36 months post-injury. Of these two longitudinal studies only one included a control group comprised of children of similar age who had sustained an orthopedic injury. 
Of the 16 studies reviewed, eight examined volumetric properties of selected brain regions only, while three considered multiple brain regions and/or all gray and white matter (see Figure 1). Two studies report on diffusion tensor imaging (DTI) in selected brain regions (corpus callosum Wilde et al., 2006 and cingulum bundle Wilde et al., 2010, respectively), while one study used DTI to examine selected white matter regions including the corpus callosum, interior capsule, superior longitudinal fasciculus and inferior fronto-occipital fasciculus (Yuan et al., 2007). Two studies employed both volumetric and DTI methods to explore evidence for impaired brain growth across the whole brain following TBI.

\section{Patient population}

Of the sixteen studies reviewed, five present various findings from a single cohort of sixteen children and youth who previously sustained a moderate-severe TBI. Six studies in total considered the moderate to severe patient population. Three studies included mild, moderate and severe case while two included "complicated mTBI" [defined as children exhibiting focal pathology on acute computed tomography (CT), regardless of having GCS scores in the range of 13-15] in addition to children and youth survivors of moderate and severe TBI. One study considered the full spectrum of TBI including mild, moderate and severe TBI. One study defined their patient population as mild to moderate and severe, while a final three studies considered severe TBI only.

\section{Behavioral outcome measures}

Just over two thirds $(11 / 16)$ of the studies correlated a measure of behavioral function with the MRI findings. A total of five studies did not include a behavioral outcome measure and three studies (Wilde et al., 2005, 2006; Yuan et al., 2007) correlated MRI findings with GCS or Glasgow Outcome Scale (GOS) alone (see Supplementary Table 1). Most studies that included a behavioral outcome measure reported positive correlations with the structural features assessed (see Figure 2). For example, Wilde

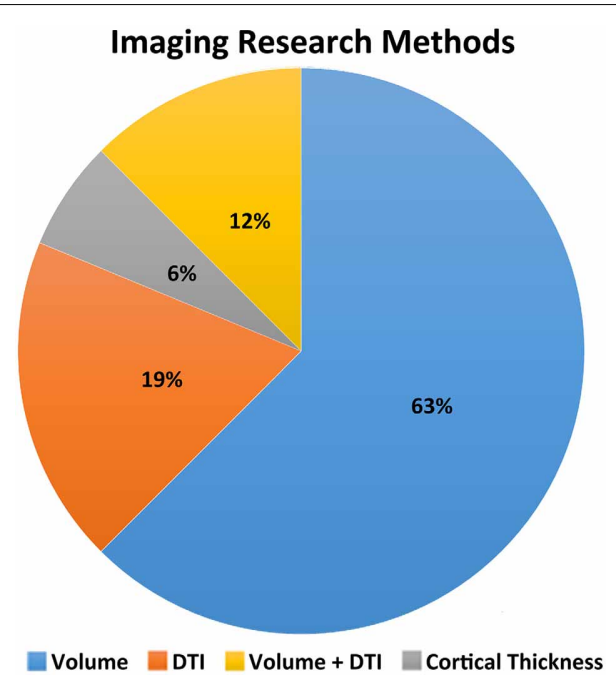

FIGURE 1 | Illustrative summary of imaging research methods. et al. (2012) found a significant positive correlation between the emotional control subscale of the Behavior Rating Inventory of Executive Function (BRIEF) and right medial frontal and right anterior cingulate gyrus volume. Levin et al. (2000) reported that the uncorrected corpus callosum area was correlated with acute TBI severity and Vineland Adaptive Behavior Scale (VABS) score at 36 months postinjury. Wilde et al. (2010) reported a significant correlation between a low GCS score and high apparent diffusion coefficient (ADC). Furthermore, for the TBI group, significant correlations were found between DTI parameters and behavioral measures. Fearing et al. (2008) found a decreased baseline RT on the Sternberg task to be associated with total brainstem volume for both the control and TBI groups. Yuan et al. (2007) found GCS scores to be positively correlated with FA in several white matter areas including the inferior frontooccipital fasciculus. Braga et al. (2007) observed lesion volume and presence of lesions left supramarginal gyrus in splenium to be significantly associated with dyscalculia. Wilde et al. (2006) found higher FA was related to increased cognitive processing speed and faster interference resolution. In the TBI patients, higher FA was also related to better functional outcome as measured by the GOS. Serra-Grabulosa et al. (2005) reported verbal long term memory to be significantly correlated with volume of cerebrospinal fluid (CSF) in the TBI group only. Hippocampal volume also correlated with visual and verbal long term recall for TBI subjects. Wilde et al. (2005) observed that greater tissue preservation predicted better recovery on the GOS. Finally, Verger et al. (2001) found that corpus callosum area strongly correlated with several measures involving processing speed and visuospatial function.

\section{CHRONIC ATROPHY AND NEURODEGENERATIVE FINDINGS Longitudinal studies}

Cortical thickness and volumetric changes. Longitudinal investigation of cortical thickness revealed that at 18 months (relative to 3 months) post-injury, bilateral frontal, fusiform, and

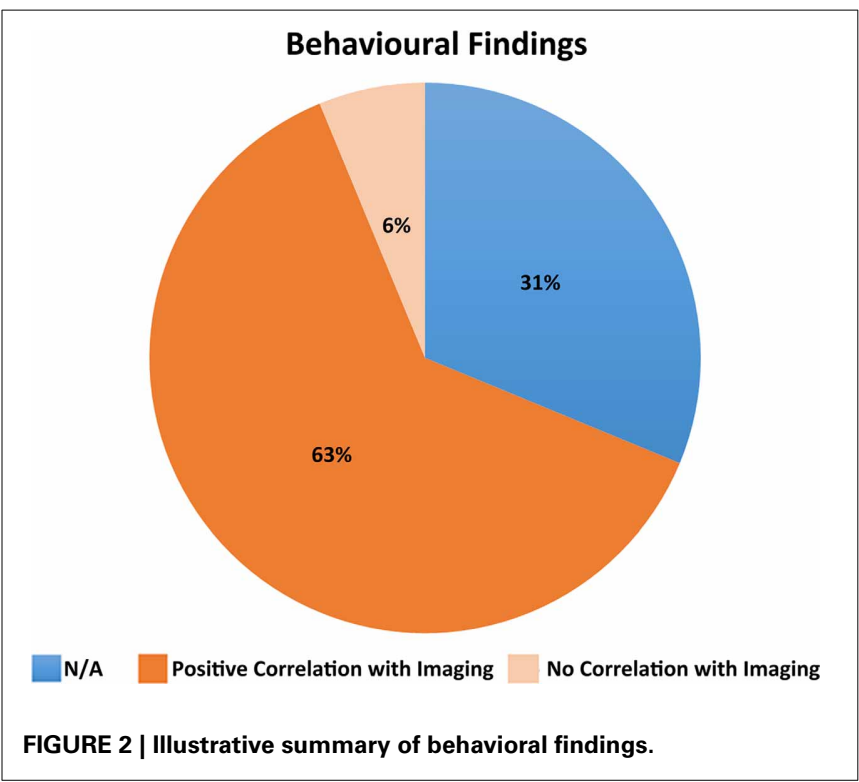


lingual regions remained significantly decreased in TBI relative to orthopedic controls with additional areas of cortical thinning emerging in bilateral frontal regions, fusiform gyrus and left parietal regions. Wilde et al. (2012) found large bilateral regions of the medial aspects of the frontal lobes and anterior cingulate were attenuated. Most notably, there were also cortical thickness increases in aspects of the medial orbital frontal lobes and bilateral cingulate and right lateral orbital frontal lobe (Wilde et al., 2012) which could be interpreted as either compensatory hypertrophy or random effects. In addition, Levin et al. (2000) report that corpus callosum area decreased from 3 to 36 months in severely injured children and increased in the mild to moderate group. Uncorrected corpus callosum area was correlated with acute TBI severity and functional outcome at 36 months post-injury.

White matter integrity assessed by diffusion tensor imaging (DTI). None of the longitudinal studies examined this parameter.

\section{Cross-sectional studies}

Cortical thickness and volumetric changes. All eleven studies focusing on volumetric changes reported positive findings indicative of long-term degeneration in selected brain regions. More specifically, relative to a control group with similar demographic characteristics and in some cases, an orthopedic or extracranial injury, volume loss was evident in the hippocampus, amygdala, globus pallidus, thalamus (gray matter only), periventricular white matter, cerebellum, and the midbrain of the brainstem. Whole brain volume was found to be significantly decreased in TBI patients relative to controls while CSF and ventricular space was observed to be significantly greater. A number of studies attempted to control for the presence of focal lesions by analyzing volumes in brain regions with no focal lesions, as well as patients who did not have focal injuries. These, studies reported reduced volumes in selected brain regions suggesting that the degeneration was not secondary to acute injury and resulting atrophy. Finally in the one study that included mTBI and stratified results according to severity (Beauchamp et al., 2011a,b), significantly reduced gray matter and left hippocampal volume was reported for mild injuries as well as significantly increased CSF compared to an age and sex matched sample.

White matter integrity assessed by diffusion tensor imaging (DTI). All five studies employing DTI reported positive findings indicating compromised white matter integrity at least 1 year following TBI compared to demographically similar control subjects, some of whom sustained an orthopedic or extracranial injury. More specifically, fractional anisotropic (FA) values were significantly reduced in the genu, body and splenium of the corpus callosum, anterior limb of the posterior capsule, posterior limb of the anterior capsule, superior fronto-occipital fasciculus, superior longitudinal fasciculus, superior fronto-occipital fasciculus, and centrum semiovale. Moreover, FA values were significantly reduced bilaterally in the cingulum bundles, while ADC values were significantly increased. Similarly, TBI patients demonstrated significantly higher mean diffusivity in the right cerebral white matter, bilaterally in the forceps major and in the body and splenium of the corpus callosum.

\section{DISCUSSION}

Taken together, the studies reviewed suggest there is evidence for long-term neurodegenerative change following TBI in children and youth. In particular both cross-sectional and longitudinal studies revealed volume loss in selected brain regions including the hippocampus, amygdale, globus pallidus, thalamus, periventricular white matter, cerebellum, and brain stem as well as overall decreased whole brain volume and increased CSF and ventricular space. DTI studies also report evidence for decreased axonal integrity, particularly in the corpus callosum (Wilde et al., 2006; Yuan et al., 2007; Porto et al., 2011). Although fewer in number, longitudinal investigations are of critical importance and those reviewed here (i.e., Wilde et al., 2012) highlight the dynamic and disruptive interplay between childhood TBI and normal developmental neuronal processes such as axonal thinning and increased myelination (see Bigler, 2013).

Taken together, the findings appear to highlight a sensitivity of the hippocampus and deep limbic structures in pediatric populations, which like adults, show similarities to CTE where there is a distinct distribution of atrophy along the amygdalo-hippocampal-septo-hypothalamic-mesencephalic continuum (McKee et al., 2009). They also corroborate findings in the chronic phase of moderate to severe TBI in adults, where sub-acute atrophy within the limbic system hippocampi (Ng et al., 2008), corpus callosum (Reeves et al., 2007), and elsewhere (i.e., Greenberg et al., 2008; Bigler et al., 2010; Green et al., 2014) have been documented.

A major limitation of the studies reviewed is the lack of studies focused specifically on repetitive concussions or mTBIs. Only one study (Beauchamp et al., 2011a,b) reported results specific to mTBI where reduced gray matter and left hippocampal volume was reported for mild injuries as well as significantly increased CSF compared to an age and sex matched sample. The second important limitation is that the methods of only a subset of the studies speak directly to a progressive, and putatively neurodegenerative entity. Chronic findings in the rest of the studies reviewed may alternatively reflect the enduring effects of the initial injuries. These findings indicate that long-term investigation of neurodegenerative change following repetitive concussions and mTBIs in children is warranted (Tartaglia et al., 2014).

There is widespread belief that children are at an advantage to adults when inflicted with significant brain damage, such as repeat concussions or mTBIs, as the developing brain has a higher chance of reorganization or plasticity (McCrory et al., 2004). This view is becoming increasingly challenged. The developing brain is cognitively maturing throughout childhood and any impact may cause a disruption in this neuronal maturation (Anderson et al., 2001). Although the injury may occur in the same way, the outcome needs to be treated differently as the composition and mechanical properties of the head and brain differ in an adult and youth (Kirkwood et al., 2006). These differences include increased brain water content, decreased level of myelination, 
skull geometry, suture elasticity, and neck strength (Bauer and Fritz, 2004; Kirkwood et al., 2006).

In conclusion, the mini-review provides strong evidence for neurodegenerative change following TBI of all severities in children and youth while clearly highlighting repetitive and chronic mTBI in children and youth as an overlooked population. Future research should employ multi-centerd strategies to longitudinally investigate the possibility of neurodegenerative onset and CTE in childhood associated with repeat mTBIs by developing age specific normal databases for each of the imaging parameters under assessment.

\section{SUPPLEMENTARY MATERIAL}

The Supplementary Material for this article can be found online at: http://www.frontiersin.org/journal/10.3389/fnhum. 2014.00139/abstract

\section{REFERENCES}

Anderson, V., Catroppa, C., Morse, S., Haritou, F., and Rosenfeld, J. (2001). Outcome from mild head injury in young children: a prospective study. J. Clin. Exp. Neuropsychol. 23, 705-717. doi: 10.1076/jcen.23.6.705.1015

Arksey, H., and O'Malley, L. (2005). Scoping studies: towards a methodological framework. Int. J. Soc. Res. Methodol. 8, 19-32. doi: $10.1080 / 1364557032000119616$

Bauer, R., and Fritz, H. (2004). Pathophysiology of traumatic injury in the developing brain: an introduction and short update. Exp. Toxicol. Pathol. 56, 65-73. doi: 10.1016/j.etp.2004.04.002

Beauchamp, M. H., Ditchfield, M., Catroppa, C., Kean, M., Godfrey, C., Rosenfeld, J. V., et al. (2011b). Focal thinning of the posterior corpus callosum: normal variant or post-traumatic? Brain Inj. 25, 950-957. doi: 10.3109/02699052.2011.589791

Beauchamp, M. H., Ditchfield, M., Maller, J. J., Catroppa, C., Godfrey, C., Rosenfeld, J. V., et al. (2011a). Hippocampus, amygdala and global brain changes 10 years after childhood traumatic brain injury. Int. J. Dev. Neurosci. 29, 137-143. doi: 10.1016/j.ijdevneu.2010.12.003

Bigler, E. D. (2013). Traumatic brain injury, neuroimaging, and neurodegeneration. Front. Hum. Neurosci. 7:395. doi: 10.3389/fnhum.2013.00395

Bigler, E. D., Abildskov, T. J., Wilde, E. A., McCauley, S. R., Li, X., Merkley, T. L., et al. (2010). Diffuse damage in pediatric traumatic brain injury: a comparison of automated versus operator-controlled quantification methods. Neuroimage 50, 1017-1026. doi: 10.1016/j.neuroimage. 2010.01 .003

Braga, L. W., Souza, L. N., Najjar, Y. J., and Dellatolas, G. (2007). Magnetic resonance imaging (MRI) findings and neuropsychological sequelae in children after severe traumatic brain injury: the role of cerebellar lesion. J. Child Neurol. 22, 1084-1089. doi: 10.1177/0883073807306246

Browne, G. J., and Lam, L. T. (2006). Concussive head injury in children and adolescents related to sports and other leisure physical activities. Br. J. Sports Med. 40, 163-168. doi: 10.1136/bjsm.2005.021220

Farbota, K. D., Bendlin, B. B., Alexander, A. L., Rowley, H. A., Dempsey, R. J., and Johnson, S. C. (2012). Longitudinal diffusion tensor imaging and neuropsychological correlates in traumatic brain injury patients. Front. Hum. Neurosci. 6:160. doi: 10.3389/fnhum.2012.00160

Fearing, M. A., Bigler, E. D., Wilde, E. A., Johnson, J. L., Hunter, J. V., Li, X., et al. (2008). Morphometric MRI findings in the thalamus and brainstem in children after moderate to severe traumatic brain injury. J. Child Neurol. 23, 729-737. doi: $10.1177 / 0883073808314159$

Green, R. E. A., Colella, B., Maller, J., Bayley, M., Glazer, J., and Mikulis, D. J. (2014). Scale and pattern of atrophy in the chronic stages of moderate-Severe traumatic brain injury. Front. Hum. Neurosci. 8:67. doi: 10.3389/fnhum.2014.00067

Greenberg, G., Mikulis, D. J., Ng, K., DeSouza, D., and Green, R. E. (2008). Use of diffusion tensor imaging to examine subacute white matter injury progression in moderate to severe traumatic brain injury. Arch. Phys. Med. Rehabil. 89, S45-S50. doi: 10.1016/j.apmr.2008.08.211
Kirkwood, M., Yeates, K., and Wilson, P. (2006). Pediatric sport-related concussion: A review of the clinical management of an oft-neglected population. Pediatrics 117, 1359-1371. doi: 10.1542/peds.2005-0994

Levin, H. S., Benavidez, D., Verger-Maestre, K., Perachio, N., Song, J., Mendelsohn, D. B., et al. (2000). Reduction of corpus callosum growth after severe traumatic brain injury in children. Neurology 54, 647-653 doi: 10.1212/WNL. 54.3.647

Martland, H. (1928). Punchdrunk. JAMA 91, 1103-1107. doi: 10.1001/jama.1928. 02700150029009

Mays, N., Roberts, E., and Popay, J. (2001). "Synthesising research evidence," in Studying the Organisation and Delivery of Health Services: Research Methods, eds N. Fulop, P. Allen, A. Clarke, and N. Black (London: Routledge), 188-220.

McCrory, P., Collie, A., Anderson, V., and Davis, G. (2004). Can we manage sport related concussion in children the same as in adults? Br. J. Sports Med. 38, 516-519. doi: 10.1136/bjsm.2004.014811

McKee, A. C., Cantu, R. C., Nowinski, C. J., Hedley-Whyte, E. T., Gavett, B. E., Budson, A. E., et al. (2009). Chronic traumatic encephalopathy in athletes: progressive tauopathy after repetitive head injury. J. Neuropathol. Exp. Neurol. 68, 709-735. doi: 10.1097/NEN.0b013e3181a9d503

Miller, H. (1966). Mental after-effects of head injury. Proc. R. Soc. Med. 59, 257-261.

Ng, K., Mikulis, D. J., Glazer, J., Kabani, N., Till, C., Greenberg, G., et al. (2008). Magnetic resonance imaging evidence of progression of subacute brain atrophy in moderate to severe traumatic brain injury. Arch. Phys. Med. Rehabil. 89, S35-S44. doi: 10.1016/j.apmr.2008.07.006

Porto, L., Jurcoane, A., Margerkurth, J., Althaus, J., Zanella, F., Hattingen, E., et al. (2011). Morphometry and diffusion MR imaging years after childhood traumatic brain injury. Eur. J. Paediatr. Neurol. 15, 493-501. doi: 10.1016/j.ejpn.2011.06.004

Reeves, T. M., Phillips, L. L., Lee, N. N., and Povlishock, J. T. (2007). Preferential neuroprotective effect of tacrolimus (FK506) on unmyelinated axons following traumatic brain injury. Brain Res. 1154, 225-236. doi: 10.1016/j.brainres.2007.04.002

Serra-Grabulosa, J. M., Junqué, C., Verger, K., Salgado-Pineda, P., Mañeru, C., and Mercader, J. M. (2005). Cerebral correlates of declarative memory dysfunctions in early traumatic brain injury. J. Neurol. Neurosurg. Psychiatry 76, 129-131. doi: 10.1136/jnnp.2004.027631

Spanos, G. K., Wilde, E. A., Bigler, E. D., Cleavinger, H. B., Fearing, M. A., Levin, H. S., et al. (2007). Cerebellar atrophy after moderate-to-severe pediatric traumatic brain injury. AJNR Am. J. Neuroradiol. 28, 537-542.

Tartaglia, M. C., Hazrati, L., Davis, K. D., Green, R. E. A., Wennberg, R., Mikulis, D., et al. (2014). Chronic traumatic encephalopathy and other neurodegenerative proteinopathies. Front. Hum. Neurosci. 8:30. doi: 10.3389/fnhum. 2014.00030

Tasker, R. C., Salmond, C. H., Westland, A. G., Pena, A., Gillard, J. H., Sahakian, B. J., et al. (2005). Head circumference and brain and hippocampal volume after severe traumatic brain injury in childhood. Pediatr. Res. 58, 302-308. doi: 10.1203/01.PDR.0000169965.08854.25

Verger, K., Junqué, C., Levin, H. S., Jurado, M. A., Pérez-Gómez, M., Bartrés-Faz, D., et al. (2001). Correlation of atrophy measures on MRI with neuropsychological sequelae in children and adolescents with traumatic brain injury. Brain Inj. 15, 211-221. doi: 10.1080/02699050010004059

Wilde, E. A., Bigler, E. D., Hunter, J. V., Fearing, M. A., Scheibel, R. S., Newsome, M. R., et al. (2007). Hippocampus, amygdala, and basal ganglia morphometrics in children after moderate-to-severe traumatic brain injury. Dev. Med. Child Neurol. 49, 294-299. doi: 10.1111/j.1469-8749. 2007.00294.x

Wilde, E. A., Chu, Z., Bigler, E. D., Hunter, J. V., Fearing, M. A., Hanten, G., et al. (2006). Diffusion tensor imaging in the corpus callosum in children after moderate to severe traumatic brain injury. J. Neurotrauma 23, 1412-1426. doi: 10.1089/neu.2006.23.1412

Wilde, E. A., Hunter, J. V., Newsome, M. R., Scheibel, R. S., Bigler, E. D., Johnson, J. L., et al. (2005). Frontal and temporal morphometric findings on MRI in children after moderate to severe traumatic brain injury. J. Neurotrauma 22, 333-344. doi: 10.1089/neu.2005.22.333

Wilde, E. A., Merkley, T. L., Bigler, E. D., Max, J. E., Schmidt, A. T., Ayoub, K. W., et al. (2012). Longitudinal changes in cortical thickness in children after traumatic brain injury and their relation to behavioral regulation and 
emotional control. Int. J. Dev. Neurosci. 30, 267-276. doi: 10.1016/j.ijdevneu. 2012.01.003

Wilde, E. A., Ramos, M. A., Yallampalli, R., Bigler, E. D., McCauley, S. R., Chu, Z., et al. (2010). Diffusion tensor imaging of the cingulum bundle in children after traumatic brain injury. Dev. Neuropsychol. 35, 333-351. doi: $10.1080 / 87565641003696940$

Yuan, W., Holland, S. K., Schmithorst, V. J., Walz, N. C., Cecil, K. M., Jones, B. V., et al. (2007). Diffusion tensor MR imaging reveals persistent white matter alteration after traumatic brain injury experienced during early childhood. AJNR Am. J. Neuroradiol. 28, 1919-1925. doi: 10.3174/ajnr.A0698

Conflict of Interest Statement: The authors declare that the research was conducted in the absence of any commercial or financial relationships that could be construed as a potential conflict of interest.
Received: 10 September 2012; accepted: 24 February 2014; published online: 19 March 2014.

Citation: Keightley ML, Sinopoli KJ, Davis KD, Mikulis DJ, Wennberg R, Tartaglia MC, Chen J-K and Tator CH (2014) Is there evidence for neurodegenerative change following traumatic brain injury in children and youth? A scoping review. Front. Hum. Neurosci. 8:139. doi: 10.3389/fnhum.2014.00139

This article was submitted to the journal Frontiers in Human Neuroscience.

Copyright () 2014 Keightley, Sinopoli, Davis, Mikulis, Wennberg, Tartaglia, Chen and Tator. This is an open-access article distributed under the terms of the Creative Commons Attribution License (CC BY). The use, distribution or reproduction in other forums is permitted, provided the original author(s) or licensor are credited and that the original publication in this journal is cited, in accordance with accepted academic practice. No use, distribution or reproduction is permitted which does not comply with these terms. 